\title{
Monte Carlo simulation-based thinning and calculating method for noninvasive blood glucose sensing by near-infrared spectroscopy
}

\author{
Congcong $\mathrm{Ma}^{*}$, Jia Qin ${ }^{\dagger}$, Qi Zhang*, Junsheng Lu*, Kexin $\mathrm{Xu}^{\star}$ \\ and Jingying Jiang,,$\S$ \\ *Tianjin Key Laboratory of Biomedical \\ Detecting Techniques and Instruments \\ College of Precision Instruments \& Opto-electronics Engineering \\ Tianjin University (TU), 92\# Weijin Road \\ Nankai District, Tianjin 300072, P. R. China \\ ${ }^{\dagger}$ San Francisco, Ophthalmology, University of California \\ 480 North Civic Drive 106 \\ Walnut Creek, California 94596, United States \\ *State Key Laboratory of Precision Measuring Technology and Instruments \\ College of Precision Instruments 83 Opto-electronics Engineering \\ Tianjin University (TU) \\ 92\# Weijin Road, Nankai District \\ Tianjin 300072, P. R. China \\ \$ingying@tju.edu.cn
}

Received 25 December 2015

Accepted 10 May 2016

Published 13 June 2016

\begin{abstract}
Previous results show that the floating reference theory (FRT) is an effective tool to reduce the influence of interference factors on noninvasive blood glucose sensing by near-infrared spectroscopy (NIRS). It is the key to measure the floating reference point (FRP) precisely for the application of FRT. Monte Carlo (MC) simulation has been introduced to quantitatively investigate the effects of positioning errors and light source drifts on measuring FRP. In this article, thinning and calculating method (TCM) is proposed to quantify the positioning error. Meanwhile, the normalization process (NP) is developed to significantly reduce the error induced by light source drift. The results according to TCM show that $7 \mu \mathrm{m}$ deviations in positioning can generate about $10.63 \%$ relative error in FRP. It is more noticeable that $1 \%$ fluctuation in light source intensity may lead to $12.21 \%$ relative errors. Gratifyingly, the proposed NP model can effectively reduce the error caused by light source drift. Therefore, the measurement system for FRPs must meet that the positioning error is less than $7 \mu \mathrm{m}$, and the light source drift is kept
\end{abstract}

$\S_{\text {Corresponding author. }}$

This is an Open Access article published by World Scientific Publishing Company. It is distributed under the terms of the Creative Commons Attribution 4.0 (CC-BY) License. Further distribution of this work is permitted, provided the original work is properly cited. 
within 1\%. Furthermore, an improvement for measurement system is proposed in order to take advantage of the NP model.

Keywords: Thinning and calculating method; Monte Carlo simulation; noninvasive blood glucose sensing; floating reference theory; positioning error; light source drift.

\section{Introduction}

Near-infrared (NIR) spectroscopy is an effective method $^{1-4}$ that assists in noninvasive and continuous monitoring of the blood glucose level. However, this method has not been widely used in clinical use since it is limited to series of factors, such as the changes of physiological background and the indeterminate experimental conditions, which may affect the signals of blood glucose to a great extent..$^{5-7}$ Here, floating reference theory (FRT $)^{4,8}$ was applied to address the aforementioned limitation. It can be pointed out that at the floating reference point (FRP), the diffuse reflection was independent of the change of blood glucose concentration. Therefore, we can take advantage of the information detected at this location as a reference signal to eliminate the unpredictable interference. Since the existence of FRP has been verified by theoretical derivation and Monte Carlo (MC) simulation in previous studies, ${ }^{3,4,8}$ the main task of putting this theory into practice is to precisely locate the FRP by experiments.

The relevant measurements have been performed with a fiber bundle detection system. ${ }^{9}$ Unavoidably, there was a deviation between the measuring and theoretical positions due to series of factors, such as positioning inaccuracy, light source drift, temperature and so on. Chen ${ }^{5}$ dedicated to finding out the errors caused by the temperature and proposed a method that could effectively reduce the influence of interference on sample spectrum. According to this model, the root mean square error of prediction (RMSEP) of glucose concentration was $17.68 \mathrm{mg} /$ dL. Min ${ }^{7}$ and Wang $^{10}$ put emphasis on developing mathematical models for modifying the influence of instrument drifts. $\mathrm{Min}^{6}$ conducted in vitro aqueous glucose solution experiments with the following three models, single-beam measurement, singlebeam measurement with reference correction and double-beam measurement with double detectors. It demonstrated that the predictive ability of the PLS model based on double-beam measurement performed more stably with an average RMSEP of $13.76 \mathrm{mg} / \mathrm{dL}$.
Errors brought by mechanical positioning inaccuracy $^{11,12}$ and light source drift ${ }^{7,10}$ may lead to mistakes in diffuse reflection spectra. However, these errors have not been quantitatively investigated in previous work. Thereby, in this manuscript, the MC simulation $^{13}$ is adopted to investigate the errors caused by the positioning and light source factors. The MC simulation ${ }^{1,13-18}$ is suitable for this research, because it provides more abundant information about the diffuse reflection light of glucose solution than those obtained from experimental observations. Herein, thinning and calculating method (TCM) is proposed and developed to quantify the positioning errors. The quantification of the error brought by positioning inaccuracy is significantly useful to understand how positioning error affect the measurement of FRP. Meanwhile, the normalization process (NP) is expressed to eliminate the errors in NIR spectra due to light source drifts. Moreover, the incorporation of NP into TCM could assist in the identification of the comprehensive effects of positioning inaccuracy and light source drift on FRP measurement. Thus, our study provides a means to improve the measurement instrument by collecting the diffuse reflection and light source intensity simultaneously, with the result of reducing the error caused by light source drift to a certain extent.

\section{Methods}

\subsection{MC simulation for the FRT}

MC simulation is a statistical model which can excellently mimic photon propagation in tissue-like media. ${ }^{1,13-19}$ It includes series of photon-tissue interactions which are dominated by optical parameters of the sample media, such as photon launch, absorption, reflection, scattering, etc. Within this study, the multi-layered media (MCML) program was previously proposed by Wang et al. ${ }^{13}$ Here, this program is employed to simulate the photon propagation in the homogeneous semi-infinite medium. 


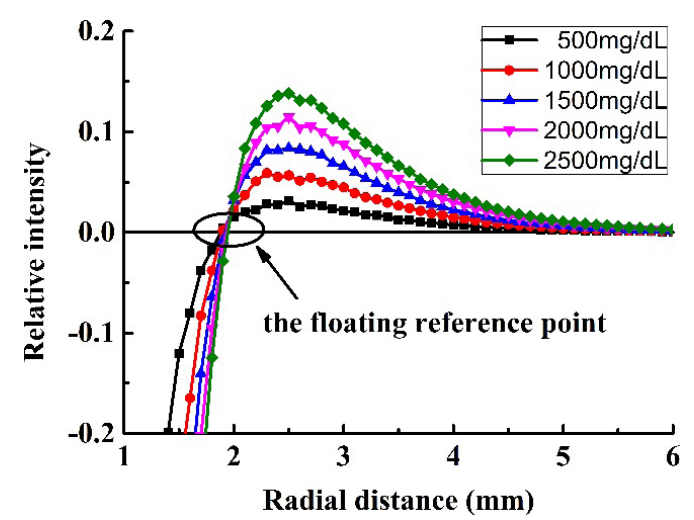

Fig. 1. The FRP of glucose in $2 \%$ Intralipid solution by simulation.

In this paper, the MC simulation is carried out with the given optical properties. ${ }^{19-25}$ The wavelength is fixed at $1310 \mathrm{~nm}$ and the photon number $(\mathrm{PN})$ is specified as $10^{10}$. The scattering medium is Intralipid solution with different glucose concentrations. The volume fraction of Intralipid is fixed at $2 \%$ and the glucose concentration ranges from 0 to $2500 \mathrm{mg} / \mathrm{dL}$, with an interval of $500 \mathrm{mg} /$ $\mathrm{dL}$. The diffused reflection signals collected from pure Intralipid solution is chosen as the reference spectrum. By deducting the reference medium, we can obtain the variations of reflection caused by glucose against with different radial distances. As shown in Fig. 1, at the wavelength of $1310 \mathrm{~nm}$, the radial FRP where the diffuse reflection is independent of the change of glucose concentration, locates at $1.93016 \mathrm{~mm}$ in $2 \%$ Intralipid solution.

\subsection{TCM for positioning inaccuracy}

The diffuse reflection signals are collected at rings according to the MCML code, and the radii of the outer circles are recorded as the radial distance (Fig. 2). The sampling rings are centered at the light source with a specified sampling interval width between each circle. Therefore, the signals detected by the rings will change with the ring areas, as well as the incident PNs. Meanwhile, the ring area is dependent on the sampling interval, thus that the diffuse reflection will be affected by the sampling interval.

The original interval $\triangle$ is $0.1 \mathrm{~mm}$ in terms of the $\mathrm{MC}$ code, so the reflection can be recorded at radial positions from 0.1 to $10 \mathrm{~mm}$ with an interval of $0.1 \mathrm{~mm}$ (Fig. 2(a)). As long as the parameter $\triangle$ is changed from 0.1 to $0.02 \mathrm{~mm}$ by uniformly dividing the original ring into five new sampling rings, we can obtain the signals at the radial positions from 0.02 to $10 \mathrm{~mm}$ with an interval of $0.02 \mathrm{~mm}$ (Fig. 2(b)).

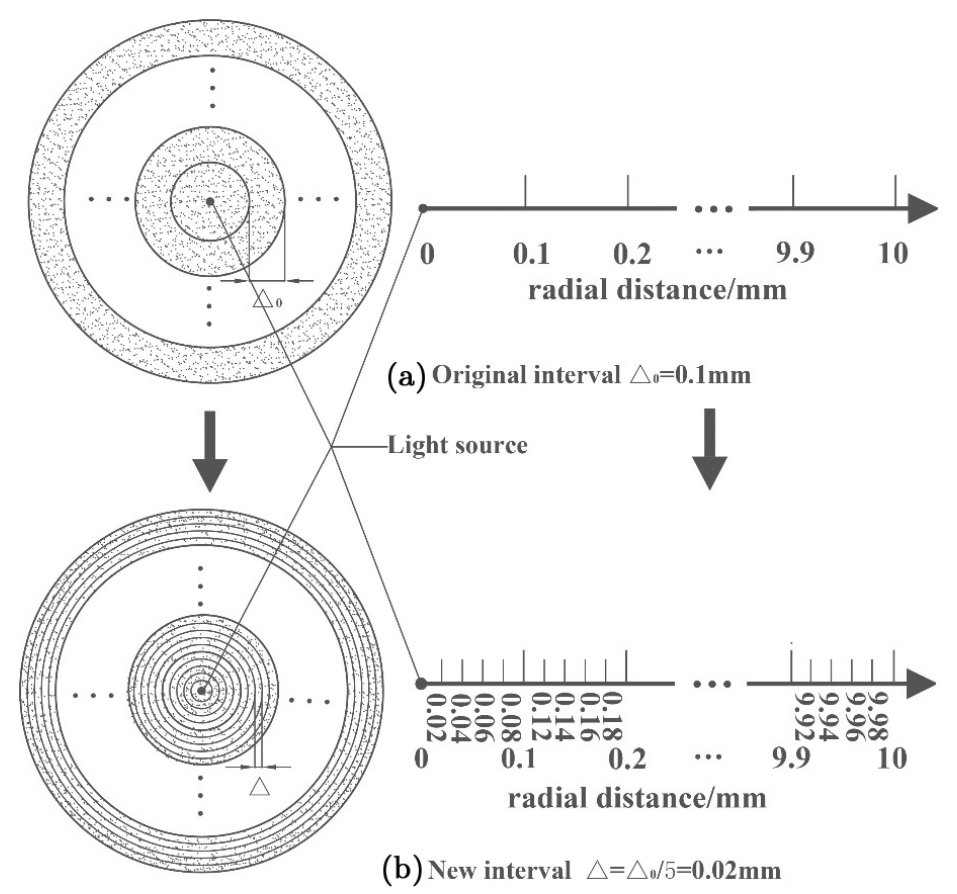

Fig. 2. Schematic of thinning the sampling interval: (a) original interval, the left represents vertical view and the right represents side view; (b) new interval, the left is vertical view and the right is side view. 
Table 1. The method of grouping the rings.

\begin{tabular}{lccccc}
\hline Group & $G 1$ & $G 2$ & $\ldots$ & $G 99$ & $G 100$ \\
\hline $\begin{array}{l}\text { Rings in the } \\
\text { group }\end{array}$ & 1st-5th & 6 th-10th & $\ldots$ & 491st-495th & 496th-500th \\
$\begin{array}{l}\text { Radial } \\
\text { distance } \\
(\mathrm{mm})\end{array}$ & 0.1 & 0.2 & $\ldots$ & 9.9 & 10 \\
\hline
\end{tabular}

Firstly, in order to analyze the influence of different intervals, the diffuse reflection light intensity is converted into $\mathrm{PN}$ as

$$
N_{i}=R d r_{i} *\left(2 \pi R_{i}\right) * \triangle * N
$$

where $N_{i}$ is the PN received at the $i$ th ring, $R d r_{i}$ is the diffused light at the $i$ th ring, $R_{i}$ is the radius at the $i$ th ring, $\triangle$ is the sampling interval and $N$ is the incident PN. The ring area can be approximated to a rectangle since it is too small. Then, the 500 new thin rings are divided into 100 groups with adjacent rings, which is listed in Table 1.

Thirdly, the PNs at radial positions are calculated by adding the PNs in corresponding groups using Eq. (2):

$$
\begin{gathered}
N_{G 1}=\sum_{i=1}^{5} N_{i}, \quad N_{G 2}=\sum_{i=6}^{10} N_{i}, \ldots, \\
N_{G 99}=\sum_{i=491}^{495} N_{i}, \quad N_{G 100}=\sum_{i=496}^{500} N_{i},
\end{gathered}
$$

where $N_{G j}(j=1,2, \ldots, 100)$ is the PNs at the $j$ th group, $N_{i}(i=1,2, \ldots, 500)$ is the PNs at the $i$ th ring.

The proximity of $N_{G}$ and $N_{o}$ (the PNs collected by the original interval) has been assessed in Table 2. Compared with $N_{o}, N_{G}$ shows excellent agreement with it.
This method, incorporating thinning, converting, grouping and calculating, is called TCM. TCM can be utilized for analyzing positioning error quantitatively.

Precisely, the original sampling ring $\triangle$ is divided into 100 new rings, and accordingly, the data sets of reflection signal are recorded at radial positions from 0.001 to $10 \mathrm{~mm}$ with an interval of $0.001 \mathrm{~mm}$. By this way, we can obtain 10,000 positions with corresponding light intensity.

Figure 3 shows the schematic of positioning inaccuracy. Point "A" represents the exact position, "B" represents the calculated position, which is closer to light source than "A", and "C" represents the calculated position, which is farther away from the light source than "A". Afterwards, we define "A" as "datum position (0)", "B" as "nearer (-)" and "C" as "farther (+)". Taking "+1" $\mu \mathrm{m}$ error for example, the 10,000 thin rings are divided into 100 groups: the first group enclose the 1st101st thin rings nearest to the light source, the last group contains the 9,902 th-10,000th thin rings farthest from the light source, and the other groups incorporate the 100 thin rings respectively in sequence. Then, the PNs at the calculated positions from 0.101 to $10.001 \mathrm{~mm}$ (with an interval of $0.1 \mathrm{~mm}$ ) can be obtained. The impact of the positioning error on measured results can be illustrated by comparing the $\mathrm{PNs}$ at the calculated position $(0.101-9.901 \mathrm{~mm})$ with that at "0" (0.1-9.9 mm). By using TCM, the detail procedures for quantitative analyses of positioning errors are described in Table 3.

Basing on the method listed in Table 3, we are able to analyze arbitrary positioning errors. Since the sampling interval can be changed to $0.5 \mu \mathrm{m}$ or even refined further, the influence of a tinier positioning error can be found out. TCM therefore

Table 2. Assessment* of the proximity of $N_{G}$ and $N_{o}$.

\begin{tabular}{lcccccc}
\hline Relative errors & $0 \mathrm{mg} / \mathrm{dL}(\%)$ & $500 \mathrm{mg} / \mathrm{dL}(\%)$ & $1000 \mathrm{mg} / \mathrm{dL}(\%)$ & $1500 \mathrm{mg} / \mathrm{dL}(\%)$ & $2000 \mathrm{mg} / \mathrm{dL}(\%)$ & $2500 \mathrm{mg} / \mathrm{dL}(\%)$ \\
\hline Max. & 7.10 & 7.06 & 7.07 & 7.08 & 7.01 & 7.16 \\
Min. & 0.02 & 0.01 & 0.03 & 0.01 & 0.01 & 0.01 \\
Mean & 1.70 & 1.71 & 1.59 & 1.67 & 1.64 & 1.70 \\
SD & 1.33 & 1.29 & 1.32 & 1.42 & 1.32 & 1.42 \\
Correlation coefficient & 99.99 & 99.99 & 99.99 & 99.99 & 99.99 & 99.99 \\
\hline
\end{tabular}

The assessment is based on the data removed the signals before $0.8 \mathrm{~mm}$. The previous studies ${ }^{2,3,7}$ has proved that FRP located farther than $1 \mathrm{~mm}$ under these parameters. 


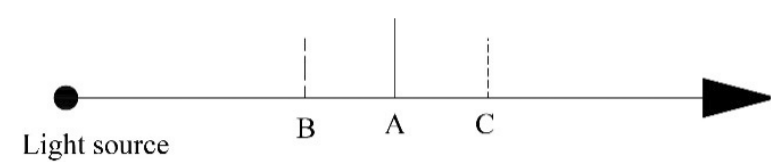

Fig. 3. Schematic of positioning inaccuracy.

becomes a more advantageous method. Firstly and most importantly, it can avoid the other error sources except the given error, resulting in more distinguishable effects of the specified errors in measurement results. Additionally, the data from one MC simulation can be used for analyzing multiple errors, which is more efficient. We cannot only introduce different magnitude errors, such as 1-10 $\mu \mathrm{m}$ (as shown in Fig. 4), but also different direction errors, such as $+5 \mu \mathrm{m},-5 \mu \mathrm{m}$ (as shown in Fig. 5).

\subsection{Light source drift}

\subsubsection{Method of analysis of light source drift based on $M C$ simulation}

In the measurement of applying the aforementioned FRT method, at least four kinds of solutions, with different glucose concentrations, need measuring for their reflection. In order to achieve more precise result, every position is required to be collected 100 times at least. The measurement ranges from 0.8 to $5 \mathrm{~mm}$, with an interval of $0.2 \mathrm{~mm}$, which means 22 positions need to be measured for one solution. Since there are four solutions, the measurement approximately lasts one hour. Therefore, it is critical to take into account the effect of light source drift during the measurement.

The light source drift has a significant effect on spectra measurement. ${ }^{6}$ In order to obtain more

Table 3. Quantitative analyses of positioning errors.

\begin{tabular}{|c|c|c|c|c|c|}
\hline $\begin{array}{l}\text { Magnitude of } \\
\text { error }(\mu \mathrm{m})\end{array}$ & +5 & +1 & 0 & -1 & -5 \\
\hline $\mathrm{PNs}$ in $G 1$ & $N_{x=0.105}=\sum_{i=1}^{105} N_{i}$ & $N_{x=0.101}=\sum_{i=1}^{101} N_{i}$ & $N_{x=0.1}=\sum_{i=1}^{100} N_{i}$ & $N_{x=0.099}=\sum_{i=1}^{99} N_{i}$ & $N_{x=0.095}=\sum_{i=1}^{95} N_{i}$ \\
\hline $\mathrm{PNs}$ in $G 2$ & $N_{x=0.205}=\sum_{i=106}^{205} N_{i}$ & $N_{x=0.201}=\sum_{i=102}^{201} N_{i}$ & $N_{x=0.2}=\sum_{i=101}^{200} N_{i}$ & $N_{x=0.199}=\sum_{i=100}^{199} N_{i}$ & $N_{x=0.195}=\sum_{i=96}^{195} N_{i}$ \\
\hline$\ldots$ & $\ldots$ & $\ldots$ & $\ldots$ & $\ldots$ & $\cdots$ \\
\hline PNs in $G 99$ & $N_{x=9.905}=\sum_{i=9,806}^{9,905} N_{i}$ & $N_{x=9.901}=\sum_{i=9,802}^{9,901} N_{i}$ & $N_{x=9.9}=\sum_{i=9,801}^{9,900} N_{i}$ & $N_{x=9.899}=\sum_{i=9,800}^{9,899} N_{i}$ & $N_{x=9.895}=\sum_{i=9,796}^{9,895} N_{i}$ \\
\hline $\begin{array}{l}\text { PNs in } G 100 \\
\quad \text { (abandoned) }\end{array}$ & $N_{x=10}=\sum_{i=9,906}^{10,000} N_{i}$ & $N_{x=10}=\sum_{i=9,902}^{10,000} N_{i}$ & $N_{x=10}=\sum_{i=9,901}^{10,000} N_{i}$ & $N_{x=9.999}=\sum_{i=9,900}^{9,999} N_{i}$ & $N_{x=9.995}=\sum_{i=9,896}^{9,995} N_{i}$ \\
\hline
\end{tabular}

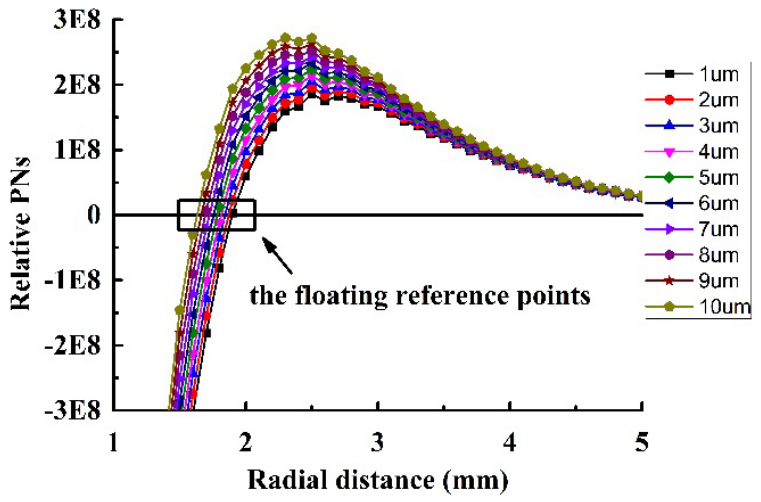

Fig. 4. The FRPs with positioning errors at different levels by simulation.

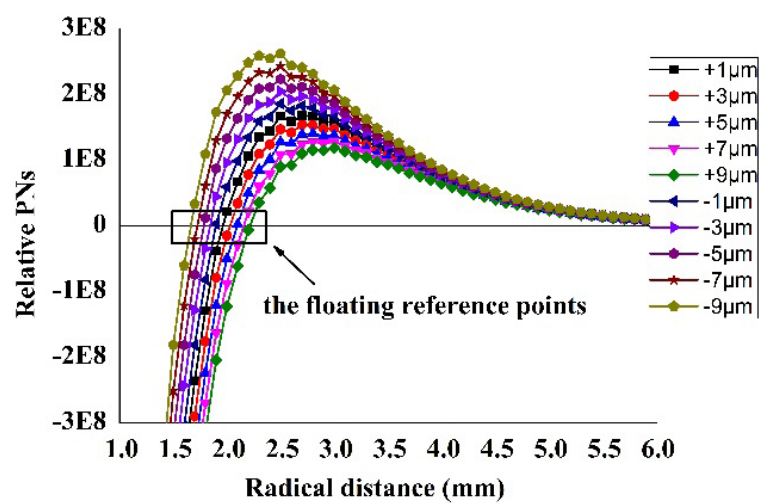

Fig. 5. The FRPs with positioning errors at different directions by simulation. 
Table 4. Error analysis method of light source drift.

\begin{tabular}{|c|c|c|c|c|c|}
\hline & Light source drift & Incident PNs & Glucose concentration $(\mathrm{mg} / \mathrm{dL})$ & Processing method & FRP \\
\hline (1) & 0 & $10^{10}$ & 0:500:2500 & $\mathrm{PN}_{\mathrm{Intra}+\operatorname{glu}_{\mathbb{\Phi}}}-\mathrm{PN}_{\mathrm{Intra}_{\mathbb{\Phi}}}$ & I \\
\hline (2) & $+1 \%$ & $1.01 * 10^{10}$ & 0:500:2500 & $\mathrm{PN}_{\text {Intra }+ \text { glu }_{\Omega}}-\mathrm{PN}_{\text {Intra }}$ & II \\
\hline (3) & $-1 \%$ & $0.99 * 10^{10}$ & 0:500:2500 & 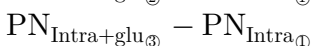 & III \\
\hline
\end{tabular}

precise measurement result, it is our aim to investigate how to identify and minimize the error originated from light source drift by using $\mathrm{MC}$ simulation modeling method. The reflection intensity obtained under $10^{10}$ incident PNs represents the situation when the light source remains stable. Meanwhile, the change of the incident PNs stochastically represents the light source drift. Through computing the differences of the reflection intensity in Intralipid solution with and without glucose, we can get the floating reference curves containing light source drift error. Compared with the curves without error, the curves with positioning error readily show the effects of source drift. The details such as the incident PNs, glucose concentration and processing methods, are described in Table 4.

There are some explanations for parameters used in Table 4. " $+1 \%$ " denotes that the incident PNs is $1 \%$ more than the reference incident PNs, and accordingly, "-1\%" denotes that the incident PNs is $1 \%$ less than the reference incident PNs; "I" means the FRP without light source drift, "II" and "III" means the FRP with " $+1 \%$ " and " $-1 \%$ " errors, respectively. The floating reference curves with different errors are shown in Fig. 6.

\subsubsection{Method of eliminating the light source drift error}

By applying the model expressed in Sec. 2.3.1 and Table 4, we can make sure that the light source drift

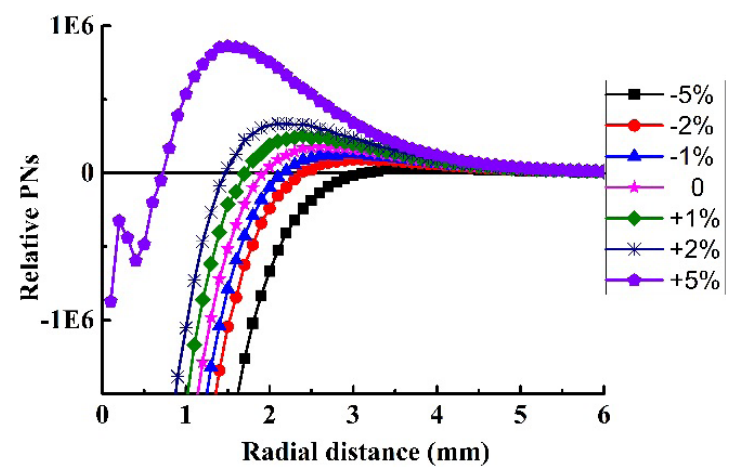

Fig. 6. The FRPs with light source drift errors by simulation. has an obvious effect on measurement of FRP. In order to eliminate the error, a NP is proposed. The main steps of NP are listed as follows:

$$
\begin{aligned}
& N_{0}=N, \\
& N_{n}=R d r_{n} *\left(2 \pi R_{n}\right) * \triangle * N, \\
& R_{n}=\frac{N_{n}}{N_{0}}=R d r_{n} *\left(2 \pi R_{n}\right) * \triangle,
\end{aligned}
$$

where $N_{0}$ equals to the incident PNs $N, N_{n}$ is the PNs of $n$th ring, and $R_{n}$ is the ratio of $N_{n}$ to $N_{0}$.

In the procedures for analyzing the light source drift introduced in Sec. 2.3.1, the difference operation is between the PNs $\left(N_{n}\right)$ of Intralipid solution with and without glucose to obtain the FRPs. NP method proposes to replace PNs $\left(N_{n}\right)$ with $R_{n}$ in the difference operations. The results are shown in Fig. 6 which indicates that the performance of NP in reducing the light source drift is satisfying.

\section{Results and Discussions}

\subsection{The effect of positioning error on FRP}

In this section, we demonstrate the simulation results of the positioning error. MC simulations were performed at $1310 \mathrm{~nm}$ with $10^{10}$ incident PNs. The simulating Intralipid solution is fixed at $2 \%$ with glucose concentration from 0 to $2500 \mathrm{mg} / \mathrm{dL}$. Previous studies ${ }^{2,3,7}$ have proved that the FRP is sensitive to wavelength and Intralipid concentration, instead of glucose concentration. Therefore, in our experiment, we use the results of Intralipid solution with glucose concentration of $2000 \mathrm{mg} / \mathrm{dL}$ as a representation. The floating reference curves with positioning errors at different levels are shown in Fig. 4 based on TCM theory.

It can be observed that the theoretical FRP locates at $1.93016 \mathrm{~mm}$ in Fig. 1, while Fig. 4 shows that an evident deviation exists between theoretical and calculated positions resulting from positioning inaccuracy. As predicted, even a tiny inaccuracy value may lead to obvious errors. The positioning 
Table 5. The error caused by different magnitude positioning inaccuracy.

\begin{tabular}{llcc}
\hline $\begin{array}{l}\text { Positioning } \\
\text { error }(\mathrm{mm})\end{array}$ & $\begin{array}{c}\text { FRP } \\
(\mathrm{mm})\end{array}$ & $\begin{array}{c}\text { Absolute } \\
\text { error }(\mathrm{mm})\end{array}$ & $\begin{array}{r}\text { Relative } \\
\text { error (\%) }\end{array}$ \\
\hline $\mathbf{0}$ & $\mathbf{1 . 9 3 0 1 6}$ & - & - \\
0.001 & 1.89636 & -0.0338 & 1.75 \\
0.002 & 1.87154 & -0.05862 & 3.04 \\
0.003 & 1.84395 & -0.08621 & 4.47 \\
0.004 & 1.81498 & -0.11518 & 5.97 \\
0.005 & 1.78588 & -0.14428 & 7.48 \\
0.006 & 1.75647 & -0.17369 & 9.00 \\
0.007 & 1.72508 & -0.20508 & 10.63 \\
0.008 & 1.69343 & -0.23673 & 12.26 \\
0.009 & 1.66422 & -0.26594 & 13.78 \\
0.01 & 1.63249 & -0.29767 & 15.42 \\
\hline
\end{tabular}

inaccuracy-dependent errors are quantitatively analyzed in Table 5.

Table 5 indicates that $1-10 \mu \mathrm{m}$ positioning errors can affect the results to a certain degree. Even $1 \mu \mathrm{m}$ inaccuracy in positioning would bring in $1.75 \%$ relative error in FRP. It is noticeable that the relative error would increase in response to the increase in positioning deviation. We can see from Table 5 that $7 \mu \mathrm{m}$ deviation in positioning leads to about $10.63 \%$ relative error in FRP.

Since the positioning error may be "nearer (-)" or "farther (+)" in actual measurements, Fig. 5 shows the effects of positioning error at different directions. Furthermore, the errors caused by different direction positioning inaccuracy are quantified in Table 6.

Figure 5 and Table 6 both further demonstrate that the positioning error does influence the FRP

Table 6 . The error caused by different direction positioning inaccuracy.

\begin{tabular}{llcc}
\hline $\begin{array}{l}\text { Positioning } \\
\text { error }(\mu \mathrm{m})\end{array}$ & $\begin{array}{c}\text { FRP } \\
(\mathrm{mm})\end{array}$ & $\begin{array}{c}\text { Absolute } \\
\text { error }(\mathrm{mm})\end{array}$ & $\begin{array}{c}\text { Relative } \\
\text { error }(\%)\end{array}$ \\
\hline+9 & 2.2146 & 0.28444 & 14.74 \\
+7 & 2.1568 & 0.22664 & 11.74 \\
+5 & 2.09586 & 0.1657 & 8.58 \\
+3 & 2.02999 & 0.09983 & 5.17 \\
+1 & 1.96326 & 0.0331 & 1.71 \\
$\mathbf{0}$ & $\mathbf{1 . 9 3 0 1 6}$ & - & - \\
-1 & 1.89636 & -0.0338 & 1.75 \\
-3 & 1.84395 & -0.08621 & 4.47 \\
-5 & 1.78588 & -0.14428 & 7.48 \\
-7 & 1.72508 & -0.20508 & 10.63 \\
-9 & 1.66422 & -0.26594 & 13.78 \\
\hline
\end{tabular}

measurement significantly. It is noteworthy to mention that different direction errors have inverse effects: the nearer error will make the theoretical location shift to a new place closer to the source, while the farther error will make a contrary effect.

According to Tables 5 and 6 , we find that the error caused by the positioning inaccuracy increases in response to the increase in positioning deviation. As long as the positioning error reaches $\pm 7 \mu \mathrm{m}$, the relative error will be more than $10 \%$. Hence, it comes to the conclusion that the positioning inaccuracy should be controlled at least in $7 \mu \mathrm{m}$ for achieving more precise measurement.

\subsection{The effect of light source drift on FRP}

In this section, we show the simulation results of light source drift. MC simulations were carried out with the parameters listed in Table 7.

By processing the spectral data based on the method expressed in Sec. 2.3.1, the curves with light source drift errors are shown in Fig. 6 (take the $2000 \mathrm{mg} / \mathrm{dL}$ solution as an illustration).

Figure 6 provides an intuitive result that the light source drift has more serious influence than the positioning inaccuracy dose. For a more credible conclusion, we analyze the error statistically and the results are listed in Table 8 .

Both Fig. 6 and Table 8 indicate that there will be a bias when the light source drift occurs during the measurement. In addition, when the source becomes stronger than that at original state, the FRP moves toward the source, vice versa.

The error caused by light source drift is more obvious than positioning inaccuracy, such as $62.54 \%$ relative error lead by $5 \%$ fluctuation. In order to eliminate this error, we adopt NP method proposed in Sec. 2.3.2.

Figure 7 shows that the error induced by light source drift has almost been eliminated, which indicates the action of NP on reducing light source error is significantly effective. The evaluation of NP is listed in Table 9 quantitatively.

According to Table 9, the NP method can significantly reduce the error caused by light source drift. The relative error have been reduced by $99.13 \%$, on average, compared with Table 8. Moreover, NP is more effective on greater light source drifts. Therefore, we can utilize the NP 
Table 7. Simulating parameters for light source drift.

\begin{tabular}{cccccc}
\hline & $\begin{array}{c}\text { Light source } \\
\text { change rate }(\%)\end{array}$ & $\begin{array}{c}\text { Radial distance } \\
(\mathrm{mm})\end{array}$ & $\begin{array}{c}\text { Wavelength } \\
(\mathrm{nm})\end{array}$ & $\begin{array}{c}\text { Intralipid } \\
\text { concentration }(\%)\end{array}$ & $\begin{array}{c}\text { Glucose concentration } \\
(\mathrm{mg} / \mathrm{dL})\end{array}$ \\
\hline (1) & -5 & $0.1: 0.1: 10$ & 1310 & 2 & $0: 500: 2500$ \\
(2) & -2 & $0.1: 0.1: 10$ & 1310 & 2 & $0: 500: 2500$ \\
(3) & -1 & $0.1: 0.1: 10$ & 1310 & 2 & $0: 500: 2500$ \\
(4) & $\mathbf{0}$ & $\mathbf{0 . 1 : 0 . 1 : 1 0}$ & $\mathbf{1 3 1 0}$ & $\mathbf{2}$ & $\mathbf{0 : 5 0 0 : 2 5 0 0}$ \\
(5) & +1 & $0.1: 0.1: 10$ & 1310 & 2 & $0: 500: 2500$ \\
(6) & +2 & $0.1: 0.1: 10$ & 1310 & 2 & $0: 500: 2500$ \\
(7) & +5 & $0.1: 0.1: 10$ & 1310 & 2 & $0: 500: 2500$ \\
\hline
\end{tabular}

Table 8. Errors of light source drift at different levels.

\begin{tabular}{llcc}
\hline $\begin{array}{l}\text { Light source } \\
\text { drift }(\%)\end{array}$ & $\begin{array}{c}\text { FRP } \\
(\mathrm{mm})\end{array}$ & $\begin{array}{c}\text { Absolute } \\
\text { error (mm) }\end{array}$ & $\begin{array}{c}\text { Relative } \\
\text { error (\%) }\end{array}$ \\
\hline-5 & 3.12674 & 1.19658 & 61.99 \\
-2 & 2.3805 & 0.45034 & 23.33 \\
-1 & 2.16574 & 0.23558 & 12.21 \\
$\mathbf{0}$ & $\mathbf{1 . 9 3 0 1 6}$ & - & - \\
+1 & 1.70553 & -0.22463 & 11.64 \\
+2 & 1.47263 & -0.45753 & 23.70 \\
+5 & 0.72304 & -1.20712 & 62.54 \\
\hline
\end{tabular}

method to reduce the error induced by light source drift.

\subsection{The effect incorporating positioning inaccuracy with light source drift on FRP}

Sections 3.1 and 3.2 have illustrated the effects of positioning inaccuracy and light source drift, respectively. In this section, we will focus on the effects at the occasion of positioning inaccuracy and

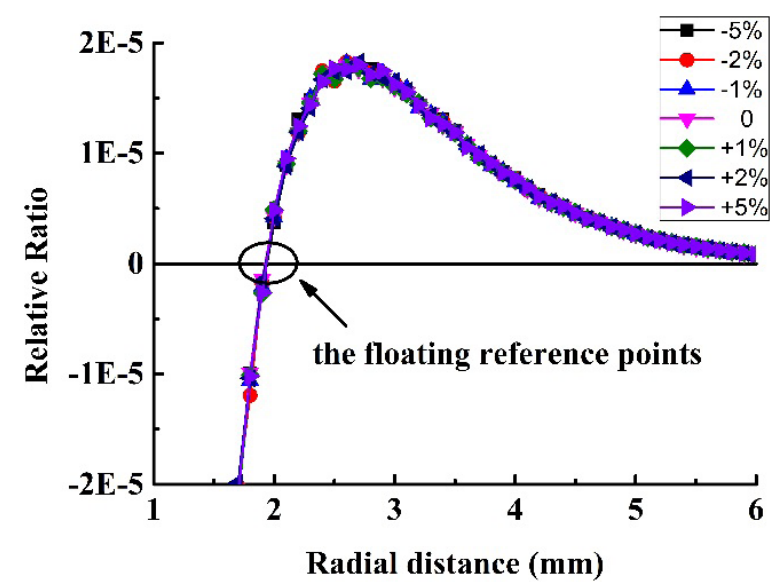

Fig. 7. The FRPs with light source drift errors with NP.
Table 9. Errors of light source drift for different levels with NP.

\begin{tabular}{lcccc}
\hline $\begin{array}{l}\text { Light source } \\
\text { drift }(\%)\end{array}$ & $\begin{array}{c}\text { FRP } \\
(\mathrm{mm})\end{array}$ & $\begin{array}{c}\text { Absolute } \\
\text { error (mm) }\end{array}$ & $\begin{array}{c}\text { Relative } \\
\text { error }(\%)\end{array}$ & $\begin{array}{c}\text { Improvement } \\
(\%)\end{array}$ \\
\hline-5 & 1.93972 & 0.00956 & 0.50 & 99.20 \\
-2 & 1.92977 & -0.00039 & 0.02 & 99.91 \\
-1 & 1.93293 & 0.00277 & 0.14 & 98.82 \\
$\mathbf{0}$ & $\mathbf{1 . 9 3 0 1 6}$ & - & - & - \\
+1 & 1.93526 & 0.0051 & 0.26 & 97.73 \\
+2 & 1.92791 & -0.00225 & 0.12 & 99.51 \\
+5 & 1.93515 & 0.00499 & 0.26 & 99.59 \\
\hline
\end{tabular}

light source drift exist simultaneously during the simulation.

Here, we mainly discuss the comprehensive effects of $\pm 10 \mu \mathrm{m}$ positioning inaccuracy and $\pm 5 \%$ light source drift. The corresponding results are shown in Fig. 8.

Compared with Figs. 5 and 6, Fig. 8(a) shows that the comprehensive errors of positioning inaccuracy and light source drift are more random and complex. The combined error is not a simple superposition of the individual effect caused by those two factors. To assess the errors more accurately, we use statistical analyses to assist our experimental analysis. The results are shown in Table 10.

Tables 6 and 8 have shown that the " + " error in positioning would make the theoretical position move away from the source and the "-" error in light source drift would make it move towards the source. However, from Table 10, we can see that the "+10 $\mathbf{\mu m} \& \mathbf{+ 5 \%}$ " error makes the theoretical location move towards the source, which indicates that the light source drift is dominantly relevant to the positioning inaccuracy. Furthermore, the NP method is adopted to reduce the light source drift and the effects of NP are presented in Fig. 8(b) and Table 11. 


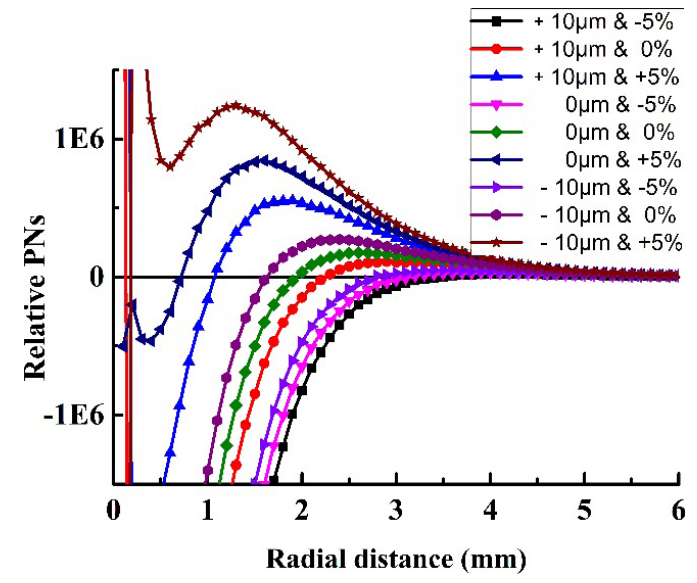

(a)

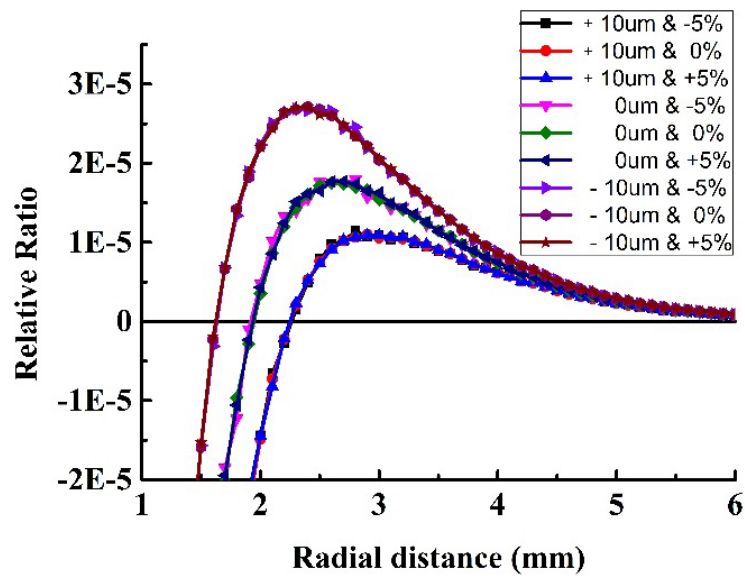

(b)

Fig. 8. The floating reference curves with positioning errors and light source drift errors: (a) without NP model and (b) with NP model.

Table 10. Comprehensive errors of positioning inaccuracy and light source drift without NP.

\begin{tabular}{lccc}
\hline $\begin{array}{l}\text { Light source } \\
\text { drift }\end{array}$ & $\begin{array}{c}\text { FRP } \\
(\mathrm{mm})\end{array}$ & $\begin{array}{c}\text { Absolute } \\
\text { error }(\mathrm{mm})\end{array}$ & $\begin{array}{c}\text { Relative } \\
\text { error (\%) }\end{array}$ \\
\hline$+10 \mu \mathrm{m} \&-5 \%$ & 3.47082 & 1.54066 & 79.82 \\
$+10 \mu \mathrm{m} \& 0 \%$ & 2.25182 & 0.32166 & 16.66 \\
$+10 \mu \mathrm{m} \&+5 \%$ & 1.07322 & -0.85694 & 44.40 \\
$0 \mu \mathrm{m} \&-5 \%$ & 3.12674 & 1.19658 & 61.99 \\
$\mathbf{0} \boldsymbol{\mu m} \& \mathbf{0 \%}$ & $\mathbf{1 . 9 3 0 1 6}$ & - & - \\
$0 \mu \mathrm{m} \&+5 \%$ & 0.72304 & -1.20712 & 62.54 \\
$-10 \mu \mathrm{m} \&-5 \%$ & 2.77475 & 0.84459 & 43.76 \\
$-10 \mu \mathrm{m} \& 0 \%$ & 1.62584 & -0.30432 & 15.77 \\
$-10 \mu \mathrm{m} \&+5 \%$ & 0.18249 & -1.74767 & 90.55 \\
\hline
\end{tabular}

It can be seen from Table 11 that NP works effectively on reducing the error caused by light source drift. Compared with Table 10, the relative error has been reduced to a certain degree after the

Table 11. Comprehensive errors of positioning inaccuracy and light source drift with NP.

\begin{tabular}{lcccc}
\hline $\begin{array}{l}\text { Light source } \\
\text { drift }\end{array}$ & $\begin{array}{c}\text { FRPs } \\
(\mathrm{mm})\end{array}$ & $\begin{array}{c}\text { Absolute } \\
\text { error (mm) }\end{array}$ & $\begin{array}{c}\text { Relative } \\
\text { error }(\%)\end{array}$ & $\begin{array}{c}\text { Improvement } \\
(\%)\end{array}$ \\
\hline$+10 \mu \mathrm{m} \&-5 \%$ & 2.26337 & 0.33321 & 17.26 & 78.37 \\
$+10 \mu \mathrm{m} \& 0 \%$ & 2.25182 & 0.32166 & 16.66 & 0.00 \\
$+10 \mu \mathrm{m} \&+5 \%$ & 2.24799 & 0.31783 & 16.47 & 62.91 \\
$0 \mu \mathrm{m} \&-5 \%$ & 1.91741 & 0.00956 & 0.50 & 99.20 \\
$\mathbf{0} \boldsymbol{\mu m}$ \& 0\% & $\mathbf{1 . 9 3 0 1 6}$ & - & - & - \\
$0 \mu \mathrm{m} \&+5 \%$ & 1.93505 & 0.00499 & 0.26 & 99.59 \\
$-10 \mu \mathrm{m} \&-5 \%$ & 1.63155 & -0.29861 & 15.47 & 64.64 \\
$-10 \mu \mathrm{m} \& 0 \%$ & 1.62584 & -0.30432 & 15.77 & 0.00 \\
$-10 \mu \mathrm{m} \&+5 \%$ & 1.62169 & -0.30847 & 15.98 & 82.35 \\
\hline
\end{tabular}

NP model has been used. Thus, NP method can be used in FRP measurement to reduce the error caused by light source drift.

\subsection{Experimental verification of the NP theory}

Based on the MC simulation, the NP theory can reduce the error caused by light source drift effectively. In this section, we will perform some experiments by using home-made measuring system to further verify the effect of the NP theory. The flow diagram of measuring system used for verification is shown in Fig. 9.

Figure 9 shows that only one fiber bundle probe has been utilized in the home-made measuring system for light transmission, which means that the spectral signal information of the glucose solution and the light source cannot be collected simultaneously during the period of FRP measuring. In order to use the NP model on the experimental results, we firstly try to shorten the time of measurement during measuring, and secondly, the light source information is obtained by putting the standard reflective plate instead of the solution after glucose solution being measured. The experiments were carried out following with the parameters sequentially listed in Table 12 .

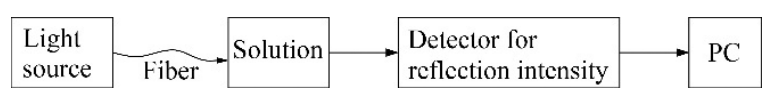

Fig. 9. Schematic of the home-made measuring system. 
Table 12. Experimental parameters for verifying NP method.

\begin{tabular}{|c|c|c|c|c|}
\hline Order & $\begin{array}{c}\text { Radial } \\
\text { distance } \\
(\mathrm{mm})\end{array}$ & $\begin{array}{l}\text { Wavelength } \\
\quad(\mathrm{nm})\end{array}$ & $\begin{array}{c}\text { Intralipid } \\
\text { concentration }\end{array}$ & $\begin{array}{c}\text { Glucose } \\
\text { concentration } \\
(\mathrm{mg} / \mathrm{dL})\end{array}$ \\
\hline (1) & $1: 0.2: 4.8$ & 1311 & $2 \%$ & 0 \\
\hline (2) & $1: 0.2: 4.8$ & 1311 & \multicolumn{2}{|c|}{ The standard reflectance plate } \\
\hline (3) & $1: 0.2: 4.8$ & 1311 & $2 \%$ & 1500 \\
\hline (4) & $1: 0.2: 4.8$ & 1311 & \multicolumn{2}{|c|}{ The standard reflectance plate } \\
\hline (5) & $1: 0.2: 4.8$ & 1311 & $2 \%$ & 2000 \\
\hline (6) & $1: 0.2: 4.8$ & 1311 & \multicolumn{2}{|c|}{ The standard reflectance plate } \\
\hline (7) & $1: 0.2: 4.8$ & 1311 & $2 \%$ & 2500 \\
\hline (8) & $1: 0.2: 4.8$ & 1311 & \multicolumn{2}{|c|}{ The standard reflectance plate } \\
\hline
\end{tabular}

Based on the spectral data measured by the system, the floating reference curves can be plotted in Fig. 10 .

Figure 10(a) shows that the FRPs measured by experiment locate dispersedly. Compared with Figs. 10(a), 10(b) shows that the points processed

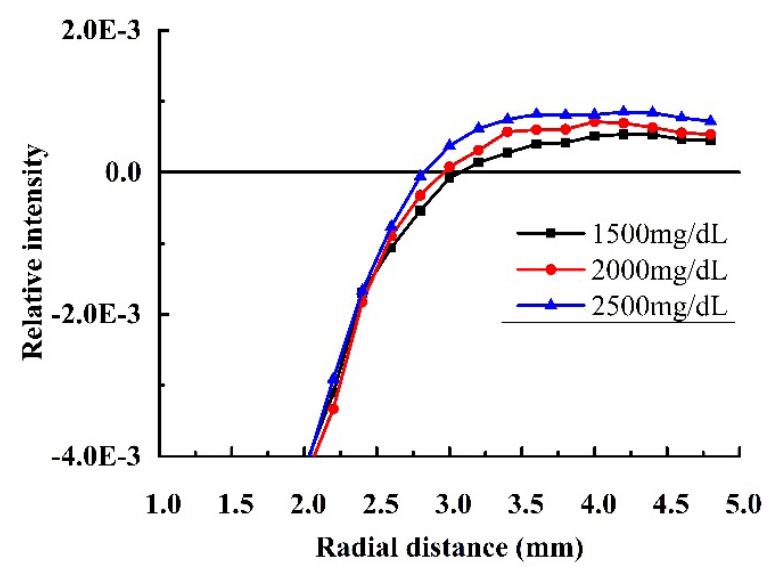

(a)

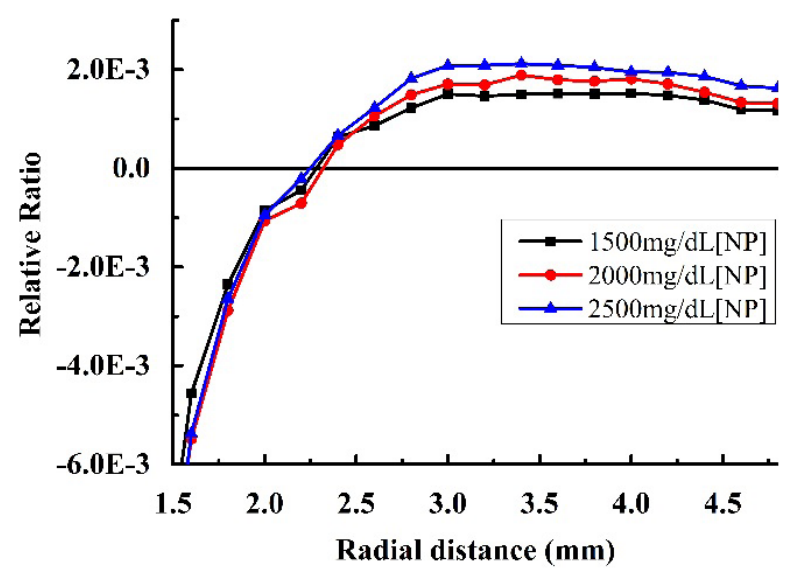

(b)

Fig. 10. The FRPs by experiment: (a) without NP model and (b) with NP model.
Table 13. Errors of light source drift of experimental measurements.

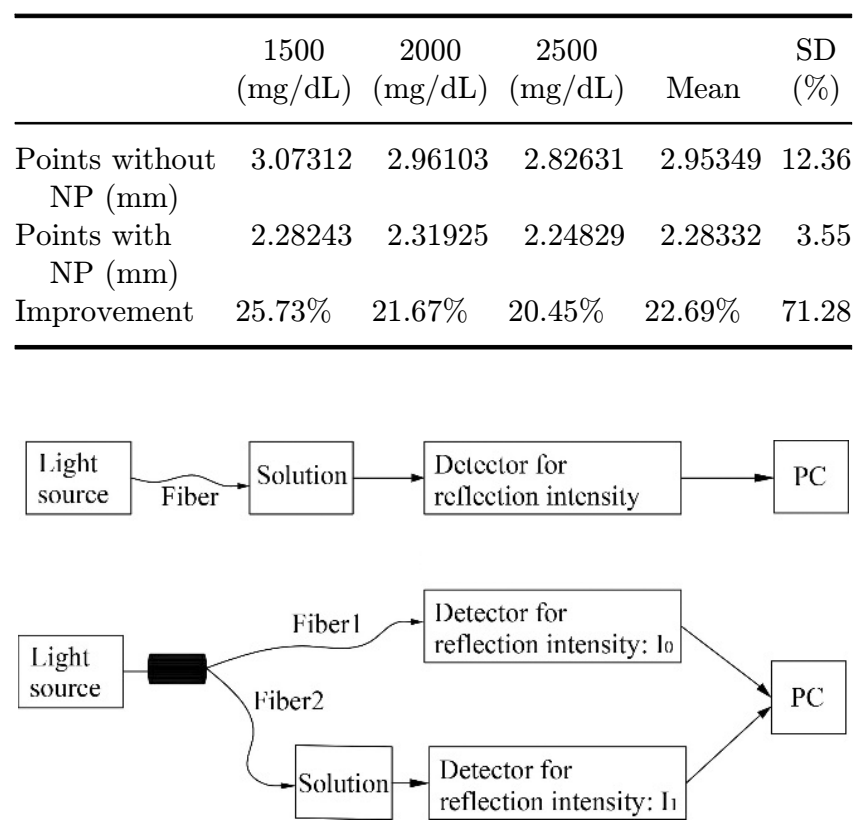

Fig. 11. Schematic of measurement system: (a) traditional measurement system and (b) improved measurement system for reducing the light source drift.

with NP is more concentrated, and the position is much closer to the MC result. Table 13 demonstrates the digital analyses based on the graphic form in Fig. 10.

According to Table 13, the measurement accuracy of the FRPs have been improved to a certain degree by NP method, and the average and standard deviation have been improved by $22.69 \%$ and $71.28 \%$, respectively.

Hence, both MC simulation and measurement experiment have proved that NP method can effectively reduce the error caused by light source drift. Thereby, a solution for improving the detection fiber of the measurement system is provided, and shown in the flow diagrams in Fig. 11.

Traditional measurement system (a) utilizes one fiber for detecting reflection intensity. Our improved system (b) utilizes two fibers for detecting both light source intensity and reflection intensity respectively, which can reduce the error induced by the light source drift using the NP method.

\section{Conclusion}

In this paper, we mainly discuss the errors caused by the positioning inaccuracy and light source drift 
based on MC simulation. Firstly, a novel method TCM is put forward to quantify the positioning error. TCM has two prominent superiorities. One is the flexibility in analyzing multiple errors on the basis of one MC simulation data, which provides the theoretical justification and improves our work efficiency. The other is the avoidance of other error sources except the specified positioning error, leading to more accurate effects of the specific error on measurement. According to the investigation based on TCM, in order to more accurately measure the FRP, the positioning error should be controlled within $7 \mu \mathrm{m}$ at least. Afterwards, the effects of light source drift are discussed quantitatively. Based on the conclusions, NP method is developed for reducing errors due to the light source drift. Moreover, this process provides a guidance to improve the measurement system by designing a fiber for collection of light intensity and reflection simultaneously. Both TCM and NP can amend the measurement of FRP so as to make the results more credible, which will improve the FRT. Thereby, both of them are very promising for assisting in in vivo noninvasive glucose sensing.

\section{Acknowledgments}

This research is performed with financial support from the National High Technology Research and Development Program of China (863 Program: 2012AA022602). This work is also supported by the 111 Project (B07014) and Tianjin Natural Science Foundation (No. 16JCZDJC31200).

\section{References}

1. M. Tarumi, M. Shimada, T. Murakami, M. Tamura, M. Shimada, H. Arimoto, Y. Yamada, "Simulation study of in vitro glucose measurement by NIR spectroscopy and a method of error reduction," Phys. Med. Biol. 48, 2373-2390 (2003).

2. F. Martelli, G. Zaccanti, "Calibration of scattering and absorption properties of a liquid diffusive medium at NIR wavelengths. CW method," Opt. Express 15(2), 486-500 (2007).

3. W. L. Chen, Z. Ma, L. An, K. X. Xu, "Applying the floating-reference method to improve the precision of noninvasive blood glucose measurement," Proc. SPIE 6445, 64450M (2007).

4. H. Yu, D. Qi, H. D. Li, K. X. Xu, W. J. Yuan, "Study on the experimental application of floating reference method to noninvasive blood glucose sensing," Spectrosc. Spect. Anal. 32, 770-774 (2012).

5. Y. Chen, Z. Z. Shi, K. X. Xu, W. L. Chen, "Study on temperature correction of near-infrared spectral of solution," Spectrosc. Spect. Anal. 29 2966-2969 (2009).

6. W. J. Zhang, R. Liu, W. Zhang, H. Jia, K. X. Xu, "Discussion on the validity of NIR spectral data in non-invasive blood glucose sensing," Biomed. Opt. Express 4, 789-802 (2013).

7. X. L. Min, R. Liu, Y. X. Hu, B. Fu, K. X. Xu, "Double-beam near-infrared spectroscopy to correct light source drift in aqueous glucose solution experiments," Anal. Methods 6, 9831-9840 (2014).

8. Y. Yang, W. L. Chen, Z. Z. Shi, K. X. Xu, "Reference point of floating-reference method for blood glucose sensing," Chin. Opt. Lett. 8, 421-424 (2010).

9. J. Y. Jiang, X. Z. Rong, H. Zhang, K. X. Xu, "A device to improve the SNR of the measurement of the positional floating reference point," Proc. SPIE 8580, 858019 (2013).

10. Z. L. Wang, W. J. Zhang, C. X. Li, W. L. Chen, $\mathrm{K} . \mathrm{X} . \mathrm{Xu}$, "The validation of the effect of correcting spectral background changes based on floating reference method by simulation," Spectrosc. Spect. Anal. 35, 547-551 (2015).

11. Y. T. Mao, Theory of Error and Precision Analysis, National Defense Industry Press, Beijing (1982).

12. Y. H. Li, Y. K. Guo, Design of Modern Precision Instruments, 2nd Edition, Tsinghua University Press, Beijing (2010).

13. L. Wang, S. L. Jacques, L. Zheng, "MCML-Monte Carlo modeling of light transport in multi-layered tissues," Comput. Meth. Prog. Bio. 47, 131-146 (1995).

14. X. W. Zhong, X. Wen, D. Zhu, "Lookup-table-based inverse model for human skin reflectance spectroscopy: Two-layered Monte Carlo simulations and experiments," Opt. Express 22(1), 1852-1864 (2014).

15. G. Zonios, A. Dimou, "Modeling diffuse reflectance from homogeneous semi-infinite turbid media for biological tissue applications: A Monte Carlo study," Opt. Express 2(12), 3284-3294 (2011).

16. S. C. Kanick, D. J. Robinson, H. J. C. M. Sterenborg, A. Amelik, "Monte Carlo analysis of single fiber reflection spectroscopy: Photon path length and sampling depth," Phys. Med. Biol. 54, 69917008 (2009).

17. X. Wen, X. W. Zhong, T. T. Yu, D. Zhu, "A Monte Carlo-based lookup table for spectrum analysis of turbid media in the reflectance probe regime," Quantum Electronics 44(7), 641-645, 2014.

18. S. F. Bish, N. Rajaram, B. Nichols, J. W. Tunnell, "Development of a noncontact diffuse optical 
spectroscopy probe for measuring tissue optical properties," J. Biomed. Opt. 16(12), 120505 (2011).

19. K. X. Xu, F. Gao, H. J. Zhao, Biomedical Photonics, 2nd Edition, Science Press, Beijing (2011).

20. T. L. Troy, S. N. Thennadil, "Optical properties of human skin in the near infrared wavelength range from 1000 to $2200 \mathrm{~nm}$, " J. Biomed. Opt. 6, 167-176 (2001).

21. G. M. Palmer, N. Ramanujam, "Monte Carlo-based inverse model for calculating tissue optical properties. Part I: Theory and validation on synthetic phantoms," Appl. Opt. 45(5), 1062-1071 (2006).

22. N. Rajaram, T. H. Nguyen, J. W. Tunnell, "Lookup table-based inverse model for determining optical properties of turbid media," J. Biomed. Opt. 13(5), 050501 (2008).

23. Z. Qian, S. S. Victor, Y. Q. Gu, C. A. Giller, H. L. Liu, "Look-Ahead Distance' of a fiber probe used to assist neurosurgery: Phantom and Monte Carlo study," Opt. Express 11(16), 1844-1855 (2003).

24. B, Luo, S. L. He, "An improved Monte Carlo diffusion hybrid model for light reflectance by turbid media," Opt. Express 15(10), 5905-5918 (2007).

25. S. L. Jacques, "Optical properties of biological tissues: A review," Phys. Med. Biol. 58, R37-R61 (2013). 